\title{
Stable ischemic heart disease: re-appraisal of coronary revascularization criteria in the light of contemporary evidence
}

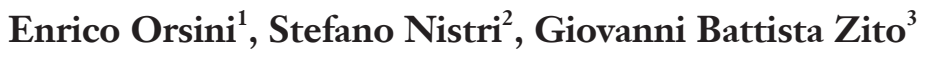 \\ ${ }^{1}$ Cardiothoracic and Vascular Department, Azienda Ospedaliera Universitaria Pisana, Pisa, Italy; ${ }^{2}$ Cardiology Service, CMSR Veneto Medica, \\ Altavilla Vicentina, Italy; ${ }^{3}$ Cardiology Service, ASL Napoli 3 Sud, Pompei, Italy \\ Contributions: (I) Conception and design: All authors; (II) Administrative support: None; (III) Provision of study materials or patients: None; (IV) \\ Collection and assembly of data: All authors; (V) Data analysis and interpretation: All authors; (VI) Manuscript writing: All authors; (VII) Final \\ approval of manuscript: All authors. \\ Correspondence to: Stefano Nistri, MD, PhD. Cardiology Service, CMSR Veneto Medica, Altavilla Vicentina, Italy. Email: stefanonistri41@gmail.com.
}

\begin{abstract}
The term "stable ischemic heart disease" includes a variety of clinical and pathophysiological situations resulting in different presentation modalities, often with complex referral patterns, and with multiple potential therapeutical options. Multifactorial pathogenesis and multiform expressivity are poorly captured by the traditional vision of ischemic heart disease (IHD) as the clogged pipes disease. The availability of different technologies for studying patients with symptoms suggestive of IHD, has shed a new light on the pathophysiology of the disease, but has also allowed appropriate follow-up of patients allocated to different therapeutical options. Though coronary revascularization has been one primary treatment option for obstructive coronary artery disease (CAD), the evidence for its efficacy in patients without acute presentation is far from optimal. A number of studies and meta-analyses strongly support the need for a personalized and optimized medical approach (including functional assessment and therapy) before the tailored option of revascularization in selected patients, in order to optimize its effects on symptoms and outcome. Most recent data have expanded the need for a more personalised approach to this complex situation, which should be patient-centered and not focused on technologies. In this review, we discuss the major pathophysiological factors and the most recent clinical data and guidelines suggestions, needed for a critical re-appraisal of the clinical decision-making to perform revascularization in patients with stable IHD. Moreover we aimed at suggesting the potential role for future studies to fill the existing knowledge gaps but also to counteract a reductive, hydraulic view of chronic IHD, which seems to be still alive and kicking, both in clinical and research communities, despite multiple evidences and recommendations.
\end{abstract}

Keywords: Stable ischemic heart disease (stable IHD); outpatients; prognosis; guidelines

Submitted Oct 02, 2019. Accepted for publication Nov 04, 2019.

doi: $10.21037 / \mathrm{cdt} .2019 .11 .02$

View this article at: http://dx.doi.org/10.21037/cdt.2019.11.02

\section{Common feeling: ischemic heart disease (IHD) as the clogged pipes disease}

IHD is traditionally perceived as the disease of the pipes clogged by fat, namely the disease caused by atherosclerotic blockages of the coronary arteries (1). In clinical practice, taken for granted the causal relationship between coronary stenosis and myocardial ischemia, the terms IHD and coronary artery disease (CAD) are indeed considered as synonyms (2). This traditional representation of IHD had its origins in the 1970s and 1980s, when researchers described the strict parallelism between the pathological evolution of coronary atherosclerosis and of its clinical manifestations. According to this model, at the stage of nonobstructive plaques would correspond the long preclinical phase of IHD, while effort angina will manifest later, for increasing volume of atherosclerotic plaques and formation of obstructive stenoses. Finally, at the end of this clinical- 


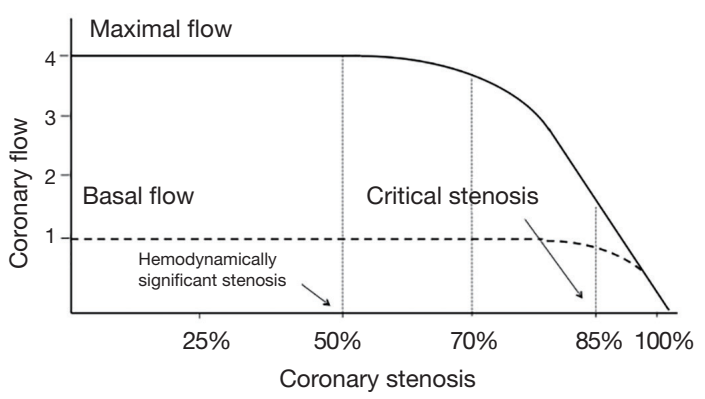

Figure 1 Relation between coronary stenosis and coronary flow in the dog. In this experimental laboratory model, Gould and Lipscomb demonstrated a direct and predictable relationship between stenosis severity and the impairment of coronary flow. Continuous line: maximal flow. Dotted line: basal flow. No effects on coronary flow has been shown for a reduction in coronary artery diameter $<50 \%$. A stenosis $\geq 50 \%$ (hemodynamically significant stenosis) limits maximal flow (coronary reserve). A stenosis $\geq 85 \%$ limits basal flow (critical stenosis). Data extrapolated from reference (5).

pathological process, acute coronary syndromes would occur due to the further evolution and complication of coronary lesions (3). In this model of IHD representation, the degree of coronary obstruction correlated with the subsequent risk of myocardial infarction (MI) (4).

The perception of a causal and necessary relationship between obstructive CAD and IHD has been supported by a series of experimental evidences from over 40 years ago, which still today underlie the modern interventional cardiology. In 1974, Gould and Lipscomb described a direct and predictable relation in the dog between the severity of coronary artery narrowing and the impairment of coronary flow (5). In this experimental laboratory model, a reduction in coronary artery diameter of $\geq 50 \%$ limited maximal coronary flow (coronary reserve), and a reduction of $\geq 85 \%$ also limited the basal flow. Since then, the stenosis $\geq 50 \%$ has been defined as hemodynamically significant coronary stenosis and the stenosis $\geq 85 \%$ as critical coronary stenosis (Figure 1).

These laboratory findings have been immediately translated in the clinical setting, where significant and critical stenoses [terms with exclusive hemodynamic meaning, of note, such as current fractional flow reserve (FFR)] automatically became ischemia-causing stenoses. According to this hydraulic model of IHD, it could be argued that there is a predictable relationship between stenosis severity and coronary flow impairment and therefore between stenosis severity and myocardial ischemia. Hence, all significant or critical stenoses should be revascularized. The availability, since the end of the 1970s, of a "simple" technique of coronary revascularization, such as coronary angioplasty (6), has encouraged the execution of an impressive number of procedures worldwide, in an attempt of treating angina and preventing $\mathrm{MI}$ and death.

Although the clogged pipes analogy and the hydraulic model of IHD are simple and evocative images for both doctors and patients, they are also wrong when applied to stable IHD, because they are not supported by scientific evidence. As outlined by Marzilli et al. in a review published in 2012 in the Journal of the American College of Cardiology (ACC), several lines of pathophysiological, epidemiological, pathological, clinical, and interventional evidences, instead, contradict this oversimplified view of stable IHD, confirming the weak link between CAD and $\operatorname{IHD}(7)$.

\section{Reality: stable IHD as a syndrome with multifactorial pathogenesis and multiform expressivity}

From a pathophysiological point of view, the direct and predictable relationship between coronary stenosis and flow, described by Gould and Lipscomb in the $\operatorname{dog}(5)$, has never been replicated in a clinical setting. In humans, conversely, an extreme variability of the ratio between stenosis severity and coronary flow has been documented, so that a $90 \%$ stenosis could be compatible with a normal coronary reserve, while a non-obstructive lesion is sometimes associated with a completely exhausted reserve (Figure 2) (8). Several factors may interfere in humans with the stenosis/ flow ratio, making it unpredictable, such as collateral circulation, coronary vasomotion and spasm, microvascular and endothelial dysfunction, transient platelet aggregation, etc. Therefore, in humans the anatomic severity of a stenosis is unable to predict its functional significance and cannot guide alone the decisions on the need for revascularization (9-11).

From an epidemiological point of view, the prevalence of stable IHD in the adult population of Western countries is about $5-6 \%$ (12), a much lower figure than the prevalence of moderate to severe coronary atherosclerosis in autopsy studies of non-cardiac deaths, which ranges from $38 \%$ in the right coronary artery to $56 \%$ in left anterior descending (LAD) coronary artery (13). Of note, $22 \%$ of the subjects in 


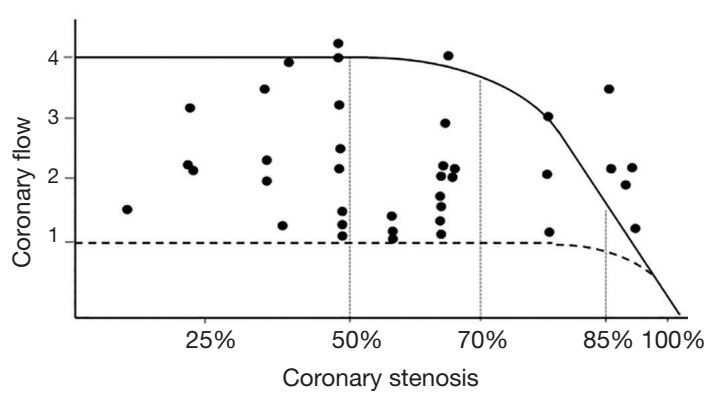

Figure 2 Relation between coronary stenosis and coronary flow in clinical setting. Continuous and dotted lines represent the direct relationship demonstrated by Gould and Lipscomb in the experimental dog. The points represent reactive hyperemic flow after 20 seconds of coronary artery occlusion in human beings, reported for coronary stenoses of different severity. In men, no predictable relationship between coronary stenosis and coronary flow can be documented. A severe stenosis (90\%) may be compatible with a normal maximal flow (coronary reserve). A nonobstructive coronary stenosis could be associated with a completely exhausted coronary reserve. Data extrapolated from reference (8).

this autopsy study had triple-vessel disease (13). Therefore, in the great majority of cases, obstructive CAD is not associated with IHD and will remain silent lifelong.

A series of clinical evidences, coming from observational studies and controlled trials, confirm the relatively low prevalence of obstructive CAD in patients with established IHD. In a large observational study by Patel et al. (14), comprising 398,978 subjects undergoing invasive coronary angiography (ICA), stratified for symptoms characteristics and results of functional tests, only $40 \%$ to $53 \%$ of the patients with typical angina had obstructive CAD, a prevalence only slightly higher than that of subjects with no symptoms $(32-43 \%)$ or atypical symptoms $(18-27 \%)$. It is also interesting to note that in this registry, the prevalence of obstructive stenoses in patients with a test positive for ischemia was not markedly different from that of those with a negative test (14). In the published trials on acute coronary syndromes, the prevalence of normal or nonobstructive coronary arteries ranged from $8 \%$ to $27 \%$ in men and from $14 \%$ to $31 \%$ in women (15). In the GUSTO IIb trial, $30.5 \%$ of women with unstable angina and $10.2 \%$ of women with STEMI had normal coronary arteries (16).

Moreover, in high-risk asymptomatic individuals the prevalence of obstructive CAD was reported to be $17 \%$ (17), and was even lower in symptomatic patients with coronary artery calcification scores of zero $(18,19)$.

This large body of evidences confirms the inconsistency of the relationship between CAD and IHD. Obstructive $\mathrm{CAD}$ is neither necessary nor sufficient for the pathogenesis of IHD because: (I) many patients with IHD do not have obstructive CAD; (II) most subjects with obstructive CAD do not have IHD.

Stable IHD is a syndrome with a complex and multifactorial pathogenesis. Pepine proposed in 2012 a pathogenetic classification of stable IHD (20), considering three main groups of causes on the basis of the location of the defect: (I) macrovascular (flow-limiting stenosis; endothelial dysfunction; coronary spasm and vasomotion; inflammation; etc.); (II) microvascular (microvascular dysfunction; endothelial dysfunction; microvascular spasm; etc.); (III) non-vascular (defects of transcellular and intracellular transport of oxygen and energy substrates and defects of mitochondrial energy production).

The multiple causes of stable IHD are not mutually exclusive, but often overlap with a variable relative role, not only in different patients, but also in the same patient, at different times of its history. The multiform phenotypic expressivity of the syndrome, from effort angina with fixed or variable threshold to mixed angina and rest angina, depends on the variable role and association of the different causes.

Syndromic features of stable IHD have been evident from decades, but only the 2019 European Society of Cardiology (ESC) Guidelines (21) have proposed the term chronic coronary syndromes (CCS), to replace the former stable CAD (2). Even with this new denomination, however, the mistake of considering IHD and CAD as synonyms still persists, leading the authors of the new guidelines to state that: "CCS are defined by the different evolutionary phases of CAD” (21).

\section{The impact of revascularization on the outcome of stable IHD}

Considering previous observations, it is not surprising that surgical or percutaneous revascularization has not been shown to improve the outcome of patients with stable IHD, with the exception, probably, of surgery in particular high-risk subgroups (large amount of ischemic or jeopardized myocardium, left main disease, 3-vessel disease with impaired left ventricular function) (2). This is easy to understand on a pathophysiological point of view, considering the weak link between stenosis and ischemia 


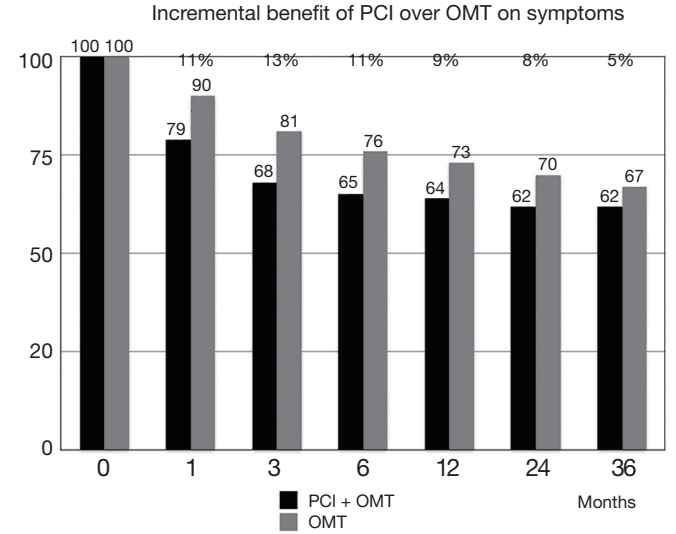

Figure 3 Persistence of symptoms during the follow-up in patients with angina at baseline in the COURAGE trial. The percentage of patients still presenting angina decreased with time in both PCI + OMT and OMT alone groups, but the incremental benefit of PCI over medical therapy progressively waned, so that at 36 months there was no significant difference between the two groups. Note that at 1 month, $79 \%$ of the patients still presented with angina after the stenosis was relieved by PCI. Data extrapolated from reference (33). OMT, optimal medical therapy; PCI, percutaneous coronary intervention.

and the possibility of other ischemic mechanisms underlying the syndrome, even in the presence of obstructive stenoses.

The failure of revascularization in affecting prognosis of stable IHD has been evident since the 1990s, with the trials of plain old balloon angioplasty, showing angina improvement but no reduction in MI or death $(22,23)$. Since then, sequential innovations in the catheter-based treatment of non-acute IHD (bare-metal stents and drugeluting stents) showed no evidence of an effect on death or MI, when compared with medical therapy (24).

In the period 2004-2015, three landmark trials, the MASS-II trial (25-27), the COURAGE trial $(28,29)$ and the BARI 2D trial (30), confirmed these pivotal evidences, showing no advantage of percutaneous coronary intervention (PCI), on top of medical therapy, on MI and death, even in patients with multivessel disease, proximal LAD disease or when the follow-up was extended up to 10-15 years.

In 2014, Stergiopoulos published a meta-analysis of trials comparing PCI on top of medical therapy $v s$. medical therapy alone in patients with stable angina and documented ischemia (31). This meta-analysis included, besides MASSII, COURAGE and BARI 2D, also the FAME 2 trial (32).
Pooling the data of the four trials, no difference was documented between PCI and medical therapy on death, MI, unplanned revascularization and angina during the follow-up. Only the FAME 2 trial showed a superiority of PCI over medical therapy on unplanned revascularizations and angina in patients with a FFR $\leq 0.80$. It is noteworthy that in the FAME 2, the up-front treatment with PCI of all patients was necessary for eliminating the need of a re-PCI in 1 of 6 patients later (32).

\section{What about angina relief?}

It is commonly reported that PCI provides more effective and incremental relief of angina in stable IHD, when compared with medical therapy $(33,34)$. While accepting this statement, however, it must be emphasized that: (I) the incremental symptoms relief wanes in a short time after PCI; (II) the superiority of PCI over medical therapy on quality of life has never been established in blinded trials, controlled by placebo.

In MASS-II (25-27), COURAGE $(28,29)$ and BARI 2D trials (30), the incremental benefit of PCI over optimal medical therapy (OMT) on symptoms was only $7 \%$ to $17 \%$ at 12 months. By 24 months in the BARI $2 \mathrm{D}$ and by 36 months in the COURAGE, freedom from angina was no longer different between PCI and OMT groups.

In the COURAGE trial $(28,29)$, the percentage of patients still presenting angina during the follow-up decreased with time in both groups, but the incremental benefit of PCI over medical therapy progressively waned, so that at 36 months there was no significant difference between groups (Figure 3). At 3 years, indeed, $38 \%$ of patients in the PCI + OMT arm and 33\% in the OMT arm alone were free from angina ( $\mathrm{P}=\mathrm{ns})$. Of note, $79 \%$ of the patients with angina at baseline in the COURAGE trial still had angina 1 month after the stenosis was relieved by a stent implantation, suggesting a different angina pathogenesis in most patients.

Moreover, all these trials evaluating the quality of life after PCI were unblinded and not controlled by placebo. Angina is a subjective symptom, influenced by the knowledge of the assigned treatment, but no study provided with methodological correctness has never been conducted in the history of angioplasty until 2018.

The impression raised by the FAME 2 trial (32), concerning the superiority of PCI over medical therapy on symptoms and quality of life, has been recently questioned by the revolutionary ORBITA trial, the first randomized, 
double-blind, placebo-controlled trial comparing exercise time and angina symptoms in patients treated with PCI + OMT or OMT alone (35). In the ORBITA, 200 patients with stable angina and single-vessel disease, were randomized after 6 weeks of medical therapy optimization to PCI or to a placebo (sham) procedure. Both the patients, the interventional cardiologists and the clinical cardiologists were not aware of the treatment assignment. After 6 weeks of follow-up, there was no significant difference between groups in exercise time increment (the primary endpoint), the physician rating of the degree of angina and the quality of life scores.

The conclusions of the authors of the ORBITA trial are shocking: "In patients with medically treated angina and severe coronary stenosis, PCI did not increase exercise time by more than the effect of a placebo procedure. The efficacy of invasive procedures can be assessed with a placebo control, as is standard for pharmacotherapy" (35).

ORBITA is a small size trial with a short follow-up time and other limitations, that does not allow definitive conclusions to be drawn. Its findings, in fact, need to be validated in larger randomized controlled trials. This trial also highlights the value of sham controls for procedureoriented studies, where a procedural placebo effect could be balanced out. Noteworthy, the physiology-stratified analysis of ORBITA, describes how blinded FFR and instantaneous wave-free ratio (iFR) values predict the placebo-controlled effect of PCI on stress echocardiography score, patientreported and physician-assessed symptoms, quality of life and treadmill exercise time. The blinded effect of PCI was more clearly seen by stress echocardiography score and freedom from angina than change in treadmill exercise time. Moreover, the lower the FFR or iFR value, the greater the magnitude of stress echocardiographic improvement caused by PCI (36).

Despite its limitations, ORBITA should be a lesson for those who uncritically believe in practices not supported by evidence.

\section{Medical treatment and coronary revascularization in stable IHD in the light of guidelines recommendations and contemporary evidence}

Both ESC $(2,21)$ and ACC/American Heart Association (AHA) $(37,38)$ guidelines on stable IHD mandate a pharmacological therapy in all patients with newly diagnosis of stable angina. Pharmacological therapy has two objectives: (I) preventing adverse cardiovascular events; (II) improving symptoms and quality of life.

Only three drugs have undoubtedly proved a favorable outcome impact in IHD: aspirin, statins and angiotensinconverting enzyme (ACE)-inhibitors. Currently available antianginal agents (beta-blockers, calcium channel blockers, nitrates, ranolazine, trimetazidine, ivabradine) have all been shown to improve to a similar extent symptoms and quality of life, but no agent has shown to prolong survival or prevent cardiovascular events, with the exception of betablockers in definite subgroups.

The 2013 ESC guidelines (2) recommend an OMT in all patients with stable IHD, defined as: "at least one drug for angina/ischemia relief plus drugs for event prevention".

As previously discussed, coronary revascularization, on top of medical therapy, confers a limited and transient symptomatic benefit, with no impact on prognosis in stable IHD, perhaps with the exception of high-risk subgroups. Of course, performing PCI or coronary artery bypass graft (CABG) on non-ischemic stenoses is not beneficial (10) and is probably harmful (11). Thus, a careful selection of ischemia- or angina-inducing stenoses is crucial to ensure benefits from revascularization.

Given this large series of observations and the disappointing results of elective revascularization, the 2013 ESC guidelines on stable IHD recommend to consider ICA for risk stratification and for selecting percutaneous or surgical revascularization, in only two subsets (2):

(I) Patients with severe stable angina (Canadian Cardiovascular Society grade 3 or 4), particularly if the symptoms are inadequately responding to medical treatment [class I, level of evidence (LOE) C];

(II) Patients with mild or no symptoms with medical therapy, in whom non-invasive risk stratification indicates a high event risk and revascularization is considered for improving prognosis (class I, LOE C).

As it is clear, the level of evidence is " $\mathrm{C}$ " (expert opinions) for both indications, lacking for this issue definitive and evidence-based conclusion.

Also the 2012 ACC/AHA guidelines on stable IHD (37), provided only two class I recommendations: (I) revascularization by means of CABG to improve prognosis in patients with left main disease or 3-vessel disease or 2 -vessel disease with involvement of proximal LAD (class I, LOE B); (II) revascularization by means of CABG or PCI to improve symptoms in patients with one or more coronary stenoses amenable to revascularization and with 
unacceptable angina despite guidelines directed medical therapy (class I, LOE A).

More recently, the 2018 ESC guidelines on myocardial revascularization (39), provided similar indications for revascularization in stable IHD: (I) for improving prognosis (left main disease; proximal LAD stenosis; two-three vessel disease and impaired left ventricular function; large area of ischemia detected by functional testing; class I, LOE A); (II) for improving symptoms (hemodynamically significant coronary artery stenosis in patients with limiting angina despite OMT; class I, LOE A). In these guidelines, clearly is stated that ascertaining the functional significance of obstructive stenoses is fundamental before deciding on revascularization.

The indications to surgical revascularization for improving prognosis in patients with severe CAD are derived from three landmark trials of the 80s and 90s [the Veteran Affairs Cooperative Study (40), the European Coronary Surgery Study (41) and the Coronary Artery Surgery Study (42)] and from a 1994 meta-analysis (43) of 7 studies that randomized a total of 2,649 patients, showing the superiority of CABG over medical therapy. Both surgical techniques and medical therapy have substantially improved in the following decades, so that it is uncertain whether the results of these pivotal trials could have been observed even nowadays.

After the disappointing results of the more recent MASS-II (25-27), COURAGE (28,29), BARI 2D (30) and FAME 2 (32) trials on the role of coronary revascularization in stable IHD, the cardiology community, and particularly the interventionists, felt that further evidence was needed. In this regard, the COURAGE trial $(28,29)$ had more detractors than supporters, criticizing the low-risk level of the population, the randomization after coronary anatomy was known, the limited use of drug-eluting stents, the high rate of crossover from OMT to PCI, etc. So that another mega-trial with similar purposes, the ISCHEMIA trial, supported by the National Heart, Lung, and Blood Institute, has been started in $2012(44,45)$. The trial is still in progress and the estimated study completion date is December 2019. However, after 7 years of recruitment have been spent, after 100 million dollars have been paid and near the end of the study and publications of the results, many experts hypothesized that the trial once again could not answer critical questions concerning the best treatment of stable IHD. In particular, criticism has raised the low recruitment rate, which has reduced the sample size than anticipated and has led to include patients with less ischemic burden.
But the main concern, which could weaken the trial results, is that the primary end-point has been recently expanded from cardiovascular death and MI to include more soft and subjective end-points, such as resuscitated cardiac arrest, hospitalization for unstable angina and hospitalization for heart failure (46). Cardiological community is asking for the need of such a change in the study methodology and inquires if the original hard end-point was not going in the desired direction. Finally, it should be emphasized that, despite the lesson of the ORBITA trial (35), ISCHEMIA is still an unblinded, not placebo-controlled trial.

\section{What is new in 2019 ESC guidelines on CCS?}

Despite the new name CCS, only few novelties are contained in the 2019 ESC guidelines (21), when compared with previous 2013 edition (2) and with 2018 ESC guidelines on myocardial revascularization (39). The features of IHD as a syndrome, for both the variety of its pathogenetic mechanisms, the polymorphism of its clinical expression and the heterogeneous outcome, have long time been established. Considering the lack of substantial new evidences from randomized trials and observational studies in the last years, the current document seems more an update, rather than a new edition of the guidelines.

Moreover, after the advent of coronary computed tomography angiography (CCTA) for the diagnosis of obstructive CAD (47), its prognostic role in the perspective of therapeutic choices is going to be elucidated. For instance, in the CONFIRM long-term registry of patients without known CAD undergoing CCTA, early revascularization is associated with reduced mortality at 5 years in patients with high-risk CAD (18). Otherwise, no benefit from early revascularization was seen in patients with low-risk CAD, while early mortality benefits in patients with intermediate-risk CAD were not sustained at 5 years (48). Furthermore, with an expanding role for CCTA in patients with stable IHD in relation to quality of life (49), a patient-centered (not methodology-centered) approach to this multi-faceted situation is needed (50).

Consistently, appropriate lifestyle management and OMT for angina/ischemia relief and for preventing vascular events, continue to be strongly recommended by current guidelines in all patients with suspected IHD. Timely review of the patient's response to medical therapy (2-4 weeks after drugs beginning) is mandatory in symptomatic patients before decisions on revascularization are taken.

Even in the current guidelines, it is recommended 
that the selection of the initial diagnostic test is based on the clinical likelihood of CAD. Coronary CTA and non-invasive functional imaging for myocardial ischemia are the preferred tests in patients with the lower to the intermediate-higher likelihood of CAD, respectively. ICA is instead recommended in patients with a very high clinical likelihood of CAD, in the presence of severe symptoms refractory to medical therapy or typical angina at low threshold, and when clinical evaluation indicates a high event risk. As in the 2018 ESC Guidelines on myocardial revascularization (39), functional assessment of stenosis significance is recommended before revascularization, unless very high grade ( $>90 \%)$ coronary artery narrowing.

Despite overall similarities with previous editions, the 2019 ESC guidelines (21) recommend a less restrictive indication for revascularization in CCS, based on the results of a recent trial (51) and two meta-analyses (52,53). However, extended follow-up of the FAME 2 trial up to 5 years (51), simply confirmed the lack of benefit of FFRguided PCI over medical therapy on MI and death, already proved at a 24-month follow-up (32). It is interesting to note that patients assigned to PCI in FAME 2 reported significantly less angina only up to 3 years of followup, while the difference with medically treated patients was no longer significant at 5 years. A very recent metaanalysis of the three randomized trials comparing FFRguided PCI with medical therapy in patients with stable coronary lesions, showed a reduction of the composite of cardiac death and MI, which was driven by a decreased risk of MI, in PCI treated patients (52). However, two-thirds of the patients included in this meta-analysis came from trials focused on non-culprit lesions of patients with STelevation MI, a very different population than stable IHD. The 2014 Windecker meta-analysis (53) is today the only evidence of a superiority of revascularization by CABG or new-generation stents, compared to medical therapy, on the survival of patients with stable IHD, with the limitations however of a Bayesian network meta-analysis.

\section{The role of CCTA in the management of stable IHD}

Current guidelines recommend ICA in patients with high pretest probability (PTP) of CAD and at least one of the following: (I) symptoms inadequately responding to medical therapy; (II) high event risk, as emerges from non-invasive evaluation (2,21,37-39). In subjects within the lower range of intermediate PTP of CAD, CCTA is considered an alternative to stress imaging techniques for ruling out stable IHD (class IIa, LOE C) (2). A second indication to CCTA, recommended by guidelines, concerns subjects with lowintermediate PTP and a non-conclusive exercise ECG or stress imaging test or who have contraindications to stress testing (class IIa, LOE C). CCTA is not recommended in patients with prior coronary revascularization (class III) and as a screening test in asymptomatic individual without clinical suspicion of IHD (class III) $(2,21)$.

Several prospective studies have established the good predictive value of CCTA in assessing not only extension and severity of obstructive CAD, but also of nonobstructive atherosclerosis (54). In the meta-analysis of Radico et al., comprising 54 studies on patients with stable angina, a relevant difference in mortality between those with non-obstructive $\mathrm{CAD}$ and those with normal coronary arteries has been observed (55). Early identification of nonobstructive CAD could therefore guide individualized preventive measures and patient therapeutic management.

Nevertheless, a series of considerations are needed when using CCTA for diagnostic or prognostic purposes: radiation exposure, a very critical issue in healthy subjects with low PTP of disease; the need of a contrast medium, potentially allergenic and nephrotoxic; the need of accurate selection of the subject (ability to hold breath, sinus rhythm, heart rate $\leq 65 \mathrm{bpm}$ ); diagnostic specificity inversely proportional to coronary calcium burden that should not exceed 400 Agatston points; low diagnostic accuracy in severe obesity; low diagnostic accuracy in patients with a coronary stent; low diagnostic accuracy, due to increase in false positives, in native coronary vessels of patients with previous CABG $(2,21)$.

The pivotal problem, however, of CCTA is that this technique provides only anatomical information on the presence, extension and severity of CAD and therefore it is not able to establish or exclude, as previously discussed, the diagnosis of IHD. The Coronary Microvascular Angina (CorMicA) randomized-controlled trial, recently demonstrated that a comprehensive approach at the time of ICA for the assessment of microvascular dysfunction and vasospastic angina in patients with non-obstructive $\mathrm{CAD}$ is superior to usual care in improving quality of life (56). The availability of techniques for diagnosing coronary artery spasm and for measuring FFR and coronary flow reserve in the catheter laboratory provides an opportunity to better ascertain the pathogenesis of ischemia, both in the presence and absence of obstructive stenoses, and therefore to guide a personalized treatment $(50,57)$. 


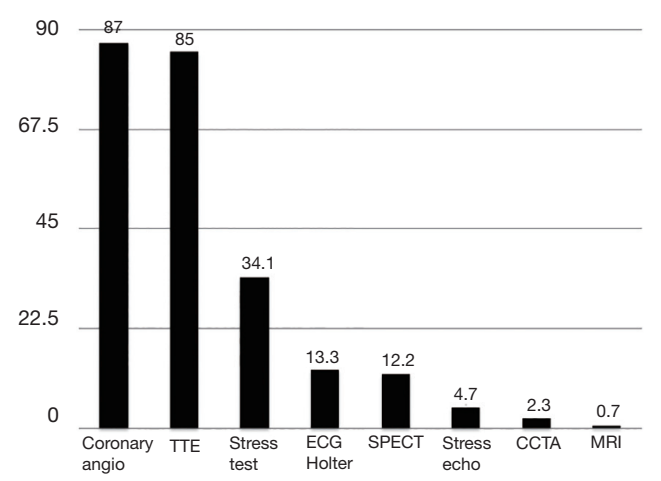

Figure 4 Diagnostic procedures performed in patients with stable IHD in the START registry. Data from reference (61). IHD, ischemic heart disease; START, STable Coronary Artery Disease RegisTry; CCTA, coronary computed tomography angiography; coronary angio, coronary angiography; MRI, magnetic resonance imaging; SPECT, stress myocardial scintigraphy; TTE, transthoracic echocardiography.

\section{Real world: when the evidence does not matter}

Despite the accumulation of evidence and the recommendations of the guidelines, reality goes in an opposite direction. In this regard, it is worth to mention the German physicist Max Planck, when he stated that "science only advances one funeral at a time". That is, when the evidence fails to persuade, for changing practices you need to replace those who practice them.

In a survey published in the Lancet in 2017, a worrying rate of inappropriateness in Italy of both coronary angiography $(30 \%)$ and coronary angioplasty (22\%) was highlighted. In this respect, Brazilian cardiologists are more appropriate than Italian cardiologists because in Brazil the rate of inappropriateness of coronary angiography has been estimated at $20 \%$. In the United States, were inappropriate procedures are not reimbursed, the rate of inappropriate PCI is markedly lower than in Italy $(1.1 \%$ for acute indications; $11.6 \%$ for non-acute indications, with variations across hospitals from $6 \%$ to $16.7 \%$ ) (58).

The problem of inappropriateness is crucial for invasive medical therapies, such as PCI, where the increase in risks adds to the increase in costs. PCI carries a little but substantial risk of complications, such as death $(0.65 \%)$, MI (15\%), renal injury (13\%), stroke $(0.2 \%)$, contrast allergy (1\%), vascular complications (2-6\%) (34). These seemingly small numbers become huge considering overall procedures performed worldwide. In Italy alone, it is estimated that between 125,000 and 150,000 PCI per year are performed (59). So, in Italy 975 deaths and 22,500 MIs would occur each year as a complication of a PCI. When $\mathrm{PCI}$ is performed in patients with stable angina, this risk is paid without a favorable impact on outcome and without a sustained incremental benefit on symptoms, in comparison with medical therapy.

Despite guideline recommendations to firstly treat all patients with an OMT, a substantial proportion of stable angina patients undergoes coronary angiography without having assessed the efficacy of an anti-anginal treatment. In a population-based study on the variations of use of OMT in stable angina before coronary angiography, only $53.6 \%$, $40.7 \%$ and $40.4 \%$ of the patients had been put on therapy with beta-blockers, calcium-channel blockers and nitrates, respectively (60).

An observational study conducted by the Associazione Nazionale Medici Cardiologi Ospedalieri (ANMCO), the STable Coronary Artery Disease RegisTry (START) registry, in which 5,070 consecutive patients were enrolled, described in 2018 the management of stable IHD in Italy (61). As it is evident from Figure 4, the vast majority of patients received a coronary angiography (87\%) and a transthoracic echocardiogram (85\%). A stress test was performed in only $34.1 \%$ of cases. Even more infrequently the patients underwent an ECG Holter (13.3\%), a myocardial scintigraphy (12.2\%) and a stress echo (4.7\%). Thus, in clinical practice, almost all patients with stable IHD receive a coronary angiography, while only a third or less undergo a functional testing to investigate the presence and the burden of myocardial ischemia. It is therefore evident that the management of stable IHD in the real world completely challenges the evidence and disattends the recommendations of the guidelines.

\section{The Arca registry for chronic angina (ARCA)}

The ARCA registry is a prospective, observational, multicentric, nationwide study projected by the Associazioni Regionali Cardiologi Ambulatoriali (A.R.C.A.) with the purpose of assessing the impact of a guidelines dictated management on quality of life and prognosis of patients with newly diagnosis of stable IHD (62).

To fully comply with guidelines recommendations, a number of information, currently not known, should be available: (I) how many patients with stable angina continue to have symptoms despite OMT? (II) Among patients with angina despite OMT, how many have coronary obstructive 


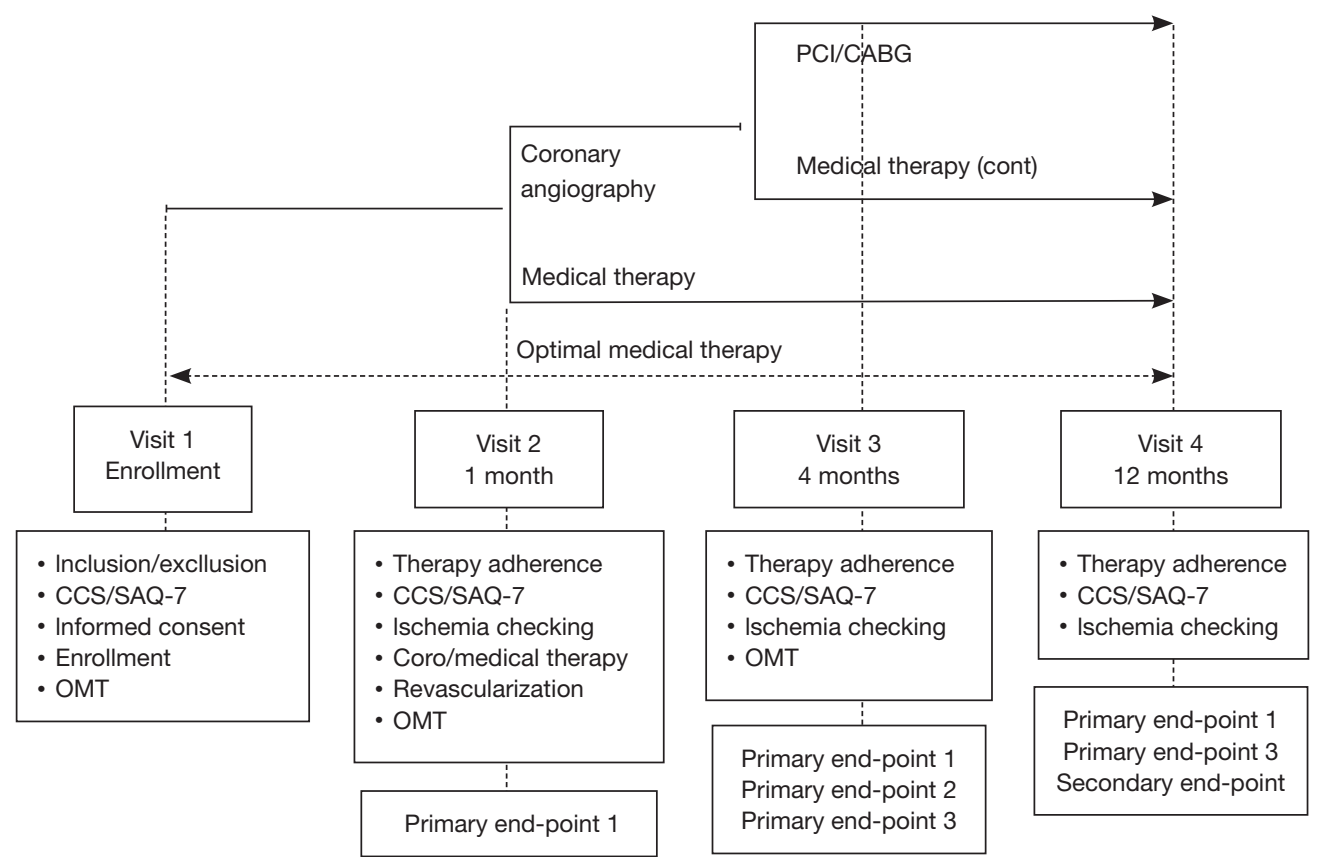

Figure 5 The flow chart of the ARCA registry study. ARCA, Arca registry for chronic angina; CABG, coronary artery bypass graft; CCS, Canadian Cardiovascular Society; OMT, optimal medical therapy; PCI, percutaneous coronary intervention; SAQ-7, Seattle Angina Quastionnaire-7.

stenoses, suitable for revascularization? (III) Among revascularized patients, how many have persistent or recurrent angina after revascularization? (IV) A treatment strategy according to guidelines recommendations, does impact on prognosis?

The flow chart of the study is illustrated in Figure 5. All patients will be firstly treated with an OMT comprising aspirin, statins, ACE-inhibitors and at least one antianginal agent. Indications for coronary angiography and revascularization or for continuing medical therapy will be given after 1 month of OMT, as recommended by current guidelines.

The end-points of the study are the following. Primary end-point 1: efficacy of OMT on angina and quality of life. Primary end-point 2: prevalence of obstructive CAD in refractory angina. Primary end-point 3: prevalence of persistent or recurrent angina after revascularization. Secondary end-point: a composite of death, MI, stroke, hospitalization for unstable angina at 1 year.

ARCA registry is still ongoing and is planned to enroll $\geq 1,000$ patients, with a follow-up of 1 year. The study is expected to be completed in 2020 .

As it is evident, ARCA registry is a profoundly different study, in the structure, the purpose and the methodology, from START registry (61). The START registry is a photograph of how stable IHD is managed in Italy. The ARCA registry is a proposal for the management of stable IHD according to the evidence and guidelines recommendations and for assessing efficacy and safety of such a management strategy.

\section{Conclusions}

Stable IHD is a syndrome with multiple pathogenetic mechanisms and multiform phenotypic expressivity. This feature has been evident from decades but only recently, last updated 2019 ESC guidelines have proposed the term CCS, to replace prior stable CAD. Even with the new denomination, the misunderstanding of believing synonyms the terms "coronary syndromes" and "ischemic syndromes" still persists. Conversely, CAD is just one of the many causes underlying chronic ischemic syndromes. About 30\% to $50 \%$ of all patients undergoing coronary angiography are found to have normal coronary artery or non-obstructive CAD. A comprehensive evaluation, both before and at the time of coronary angiography, should be made for identifying specific pathogenetic mechanisms responsible for the individual ischemic syndrome, in order to establish a 
personalized treatment and to improve its management.

It has been consistently reported that revascularization does not impact prognosis and only temporarily improves symptoms in patients with stable IHD. Therefore, contemporary evidence and current guidelines support coronary revascularization only in high-risk patients and in those with unacceptable angina despite medical therapy. Doctors should inform their patients about the uncertainty of the benefits of revascularization, so that they can share the decisions on the best treatment options.

As demonstrated by the START registry (61), the management of stable IHD in the real world goes instead in opposite directions, probably because the evidence accumulated over the last decades still fails to convince cardiological community. Cardiologists need no new names for IHD but rather new certainties. The results of studies such as ISCHEMIA (44) and ARCA Registry (62), despite their diversity, are therefore necessary. ARCA registry, in particular, is a further attempt to persuade cardiologists that the evidence does matter and to challenge Max Planck, when he stated that "science only advances one funeral at a time".

\section{Acknowledgments}

Funding: None.

\section{Footnote}

Provenance and Peer Review: This article was commissioned by the Guest Editor (Filippo Cademartiri) for the series "Clinical Impact of Cardiac CT in Clinical Practice" published in Cardiovascular Diagnosis and Therapy. The article was sent for external peer review organized by the Guest Editor and the editorial office.

Conflicts of Interest: All authors have completed the ICMJE uniform disclosure form (available at http://dx.doi. org/10.21037/cdt.2019.11.02). The series "Impact of Cardiac CT in Clinical Practice" was commissioned by the editorial office without any funding or sponsorship. The authors have no other conflicts of interest to declare.

Ethical Statement: The authors are accountable for all aspects of the work in ensuring that questions related to the accuracy or integrity of any part of the work are appropriately investigated and resolved.
Open Access Statement: This is an Open Access article distributed in accordance with the Creative Commons Attribution-NonCommercial-NoDerivs 4.0 International License (CC BY-NC-ND 4.0), which permits the noncommercial replication and distribution of the article with the strict proviso that no changes or edits are made and the original work is properly cited (including links to both the formal publication through the relevant DOI and the license). See: https://creativecommons.org/licenses/by-nc-nd/4.0/.

\section{References}

1. Rothberg MB. Coronary artery disease as clogged pipes. A misconceptual model. Circ Cardiovasc Qual Outcomes 2013;6:129-32.

2. Task Force Members, Montalescot G, Sechtem U, et al. 2013 ESC guidelines on the management of stable coronary artery disease. Eur Heart J 2013;34:2949-3003.

3. Libby P, Theroux P. Pathophysiology of coronary artery disease. Circulation 2005;111:3481-8.

4. Proudfit WL, Bruschke AV, Sones FM Jr. Natural history of obstructive coronary artery disease: ten-year study of 601 nonsurgical cases. Prog Cardiovasc Dis 1978;21:53-78.

5. Gould KL, Lipscomb K. Effects of coronary stenoses on coronary flow reserve and resistance. Am J Cardiol 1974;34:48-55.

6. Hurst JW. The first coronary angioplasty as described by Andreas Gruentzig. Am J Cardiol 1986;57:185-6.

7. Marzilli M, Merz CN, Boden WE, et al. Obstructive coronary atherosclerosis and ischemic heart disease: an elusive link! J Am Coll Cardiol 2012;60:951-6.

8. White CW, Creighton BW, Doty DB, et al. Does visual interpretation of the coronary arteriogram predict the physiologic importance of a coronary stenosis? N Engl J Med 1984;310:819-24.

9. Marcus ML, Harrison DG, White CW, et al. Assessing the physiological significance of coronary obstruction in man. Can J Cardiol 1986;Suppl A:195A-9A.

10. Bech GJ, De Bruyne B, Pijls NH, et al. Fractional flow reserve to determine the appropriateness of angioplasty in moderate coronary stenosis: a randomized trial. Circulation 2001;103:2928-34.

11. Tonino PA, De Bruyne B, Pijls NH, et al. Fractional flow reserve versus angiography for guiding percutaneous coronary intervention. N Engl J Med 2009;360:213-24.

12. Writing Group Members, Mozaffarian D, Benjamin EJ, et al. Executive summary: heart disease and stroke 
statistics--2016 update: a report from the American Heart Association. Circulation 2016;133:447-54.

13. Vyas P, Gonsai RN, Meenakshi C, et al. Coronary atherosclerosis in noncardiac deaths: an autopsy study. J Midlife Health 2015;6:5-9.

14. Patel MR, Peterson ED, Dai D, et al. Low diagnostic yield of elective coronary angiography. N Engl J Med 2010;362:886-95.

15. Anderson RD, Pepine CJ. Gender differences in the treatment for acute myocardial infarction: bias or biology? Circulation 2007;115:823-6.

16. Hochman JS, Tamis JE, Thompson TD, et al. Sex, clinical presentation and outcome in patients with acute coronary syndromes. Global use of strategies to open occluded coronary arteries in acute coronary syndromes $\mathrm{IIb}$ investigators. N Engl J Med 1999;341:226-32.

17. Cademartiri F, Maffei E, Palumbo A, et al. Coronary calcium score and computed tomography coronary angiography in high-risk asymptomatic subjects: assessment of diagnostic accuracy and prevalence of non-obstructive coronary artery disease. Eur Radiol 2010;20:846-54.

18. Villines TC, Hulten EA, Shaw LJ, et al. Prevalence and severity of coronary artery disease and adverse events among symptomatic patients with coronary artery calcification scores of zero undergoing coronary computed tomography angiography: results from the CONFIRM (coronary CT angiography evaluation for clinical outcomes: an international multicenter) registry. J Am Coll Cardiol 2011;58:2533-40.

19. Mittal TK, Pottle A, Nicol E, et al. Prevalence of obstructive coronary artery disease and prognosis in patients with stable symptoms and a zero coronary calcium score. Eur Heart J Cardiovasc Imaging 2017;18:922-9.

20. Pepine CJ, Douglas PS. Rethinking stable ischemic heart disease: is this the beginning of a new era? J Am Coll Cardiol 2012;60:957-9.

21. Knuuti J, Wijns W, Saraste A, et al. 2019 ESC guidelines for the diagnosis and management of chronic coronary syndromes. Eur Heart J 2020;41:407-77.

22. Parisi AF, Folland ED, Hartigan P. A comparison of angioplasty with medical therapy in the treatment of single-vessel coronary artery disease. Veterans affairs ACME investigators. N Engl J Med 1992;326:10-6.

23. Coronary angioplasty versus medical therapy for angina: the second randomised intervention treatment of angina (RITA-2) trial. RITA-2 trial participants. Lancet 1997;350:461-8.
24. Trikalinos TA, Alsheikh-Ali AA, Tatsioni A, et al. Percutaneous coronary interventions for non-acute coronary artery disease: a quantitative 20-year synopsis and a network meta-analysis. Lancet 2009;373:911-8.

25. Hueb W, Soares PR, Gersh BJ, at al. The medicine, angioplasty, or surgery study (MASS-II): a randomized, controlled clinical trial of three therapeutic strategies for multivessel coronary artery disease: one-year results. J Am Coll Cardiol 2004;43:1743-51.

26. Hueb W, Lopes N, Gersh BJ, et al. Five-year follow-up of the medicine, angioplasty, or surgery study (MASSII): a randomized, controlled clinical trial of 3 therapeutic strategies for multivessel coronary artery disease. Circulation 2007;115:1082-9.

27. Hueb W, Lopes N, Gersh BJ, et al. Ten-year follow-up survival of the medicine, angioplasty, or surgery study (MASS II): a randomized controlled clinical trial of 3 therapeutic strategies for multivessel coronary artery disease. Circulation 2010;122:949-57.

28. Boden WE, O'Rourke RA, Teo KK, et al. Optimal medical therapy with or without PCI for stable coronary disease. N Engl J Med 2007;356:1503-16.

29. Sedlis SP, Hartigan PM, Teo KK, et al. Effect of PCI on long-term survival in patients with stable ischemic heart disease. N Engl J Med 2015;373:1937-46.

30. BARI 2D Study Group, Frye RL, August P, et al. A randomized trial of therapies for type 2 diabetes and coronary artery disease. N Engl J Med 2009;360:2503-15.

31. Stergiopoulos K, Boden WE, Hartigan P, et al.

Percutaneous coronary intervention outcomes in patients with stable obstructive coronary artery disease and myocardial ischemia: a collaborative meta-analysis of contemporary randomized clinical trials. JAMA Intern Med 2014;174:232-40.

32. De Bruyne B, Pijls NH, Kalesan B, et al. Fractional flow reserve-guided PCI versus medical therapy in stable coronary disease. N Engl J Med 2012;367:991-1001.

33. Weintraub WS, Spertus JA, Kolm P, et al. Effect of PCI on quality of life in patients with stable coronary disease. N Engl J Med 2008;359:677-87.

34. Mitchell JD, Brown DL. Harmonizing the paradigm with the data in stable coronary artery disease: a review and viewpoint. J Am Heart Assoc 2017. doi: 10.1161/ JAHA.117.007006.

35. Al-Lamee R, Thompson D, Dehbi H-M, et al. Percutaneous coronary intervention in stable angina (ORBITA): a double-blind, randomised controlled trial. Lancet 2018;391:31-40. 
36. Al-Lamee R, Howard JP, Shun-Shin MJ, et al. Fractional flow reserve and instantaneous wave-free ratio as predictors of the placebo-controlled response to percutaneous coronary intervention in stable single-vessel coronary artwery disease. Circulation 2018;138:1780-92.

37. Fihn SD, Gardin JM, Abrams J, et al. 2012 ACCF/ AHA/ACP/AATS/PCNA/SCAI/STS guideline for the diagnosis and management of patients with stable ischemic heart disease: a report of the American College of Cardiology Foundation/American Heart Association task force on practice guidelines, and the American College of Physicians, American Association for Thoracic Surgery, Preventive Cardiovascular Nurses Association, Society for Cardiovascular Angiography and Interventions, and Society of Thoracic Surgeons. Circulation 2012;126:e354-471.

38. Fihn SD, Blankenship JC, Alexander KP, et al. 2014 ACC/AHA/AATS/PCNA/SCAI/STS focused update of the guideline for the diagnosis and management of patients with stable ischemic heart disease: a report of the American College of Cardiology/American Heart Association Task Force on Practice Guidelines, and the American Association for Thoracic Surgery, Preventive Cardiovascular Nurses Association, Society for Cardiovascular Angiography and Interventions, and Society of Thoracic Surgeons. Circulation 2014;130:1749-67.

39. Neumann FJ, Sousa-Uva M, Ahlsson A, et al. 2018 ESC/ EACTS guidelines on myocardial revascularization. Eur Heart J 2019;40:87-165.

40. VA Coronary Artery Bypass Surgery Cooperative Study Group. Eighteen-year follow-up in the Veterans Affairs Cooperative Study of coronary artery bypass surgery for stable angina. Circulation 1992;86:121-30.

41. Varnauskas E. Twelve-year follow-up of survival in the randomized European Coronary Surgery Study. N Engl J Med 1988;319:332-7.

42. Passamani E, Davis KB, Gillespie MJ, et al. A randomized trial of coronary artery bypass surgery. Survival of patients with a low ejection fraction. N Engl J Med 1985;312:1665-71.

43. Yusuf S, Zucker D, Peduzzi P, et al. Effect of coronary artery bypass graft surgery on survival: overview of 10year results from randomised trials by the Coronary Artery Bypass Graft Surgery Trialists Collaboration. Lancet 1994;344:563-70.

44. International study of comparative health effectiveness with medical and invasive approaches (ISCHEMIA).
ClinicalTrials.gov Identifier: NCT01471522. Available online: https://clinicaltrials.gov/ct2/show/NCT01471522

45. ISCHEMIA Trial Research Group, Maron DJ, Hochman JS, et al. International study of comparative health effectiveness with medical and invasive approaches (ISCHEMIA) trial: rationale and design. Am Heart J 2018;201:124-35.

46. O'Riordan M. Once again, ISCHEMIA trial feels the squeeze. 2018. Available online: https://www.tctmd.com/ news/once-again-ischemia-trial-feels-squeeze

47. Haase R, Schlattmann P, Gueret P, et al. Diagnosis of obstructive coronary artery disease using computed tomography angiography in patients with stable chest pain depending on clinical probability and in clinically important subgroups: meta-analysis of individual patient data. BMJ 2019;365:11945.

48. Schulman-Marcus J, Lin FY, Gransar H, et al. Coronary revascularization vs. medical therapy following coronarycomputed tomographic angiography in patients with low-, intermediate-, and high-risk coronary artery disease: results from the CONFIRM long-term registry. Eur Heart J Cardiovasc Imaging 2017;18:841-8.

49. Williams MC, Hunter A, Shah A, et al. Symptoms and quality of life in patients with suspected angina undergoing CT coronary angiography: a randomised controlled trial. Heart 2017;103:995-1001.

50. Cademartiri F, Nistri S, Tarantini G, et al. Management of coronary artery disease with cardiac CT beyond gatekeeping. Heart 2017;103:975-6.

51. Xaplanteris P, Fournier S, Pijls NHJ, et al. Five-year outcomes with PCI guided by fractional flow reserve. N Engl J Med 2018;379:250-9.

52. Zimmermann FM, Omerovicd E, Fournier S, et al. Fractional flow reserve-guided percutaneous coronary intervention vs. medical therapy for patients with stable coronary lesions: meta-analysis of individual partient data. Eur Heart J 2019;40:180-6.

53. Windecker S, Stortecky S, Stefanini GG, et al. Revascularisation versus medical treatment in patients with stable coronary artery disease: network meta-analysis. BMJ 2014;348:g3859.

54. Hulten EA, Carbonaro S, Petrillo SP, et al. Prognostic value of cardiac computed tomography angiography: a systematic review and meta-analysis. J Am Coll Cardiol 2011;57:1237-47.

55. Radico F, Zimarino M, Fulgenzi F, et al. Determinants of the long-term clinical outcomes in patients with angina without obstructive coronary artery disease: a systematic 
review and meta-analysis. Eur Heart J 2018;39:2135-46.

56. Ford TJ, Stanley B, Good R, et al. Stratified medical therapy using invasive coronary function testing in angina: the CorMicA trial. J Am Coll Cardiol 2018;72:2841-55.

57. Rahman H, Corcoran D, Aetesam-Ur-Rahman M, et al. Diagnosis of patients with angina and non-obstructive coronary disease in the catheter laboratory. Heart 2019;105:1536-42.

58. Brownlee S, Chalkidou K, Doust J, et al. Evidence for overuse of medical services around the world. Lancet 2017;390:156-68.

59. Berti S, Piccaluga E, Marchese A, et al. Documento di posizione SICI-GISE sugli standard e linee guida per i laboratori di diagnostica e interventistica cardiovascolare. G Ital Cardiol 2015;16:590-600.

60. Oxner A, Elbaz-Greener G, Qui F, et al. Variations in use

Cite this article as: Orsini E, Nistri S, Zito GB. Stable ischemic heart disease: re-appraisal of coronary revascularization criteria in the light of contemporary evidence. Cardiovasc Diagn Ther 2020;10(6):1992-2004. doi: 10.21037/cdt.2019.11.02 of optimal medical therapy in patients with nonobstructive coronary artery disease: a population-based study. J Am Heart Assoc 2017. doi: 10.1161/JAHA.117.007526.

61. De Luca L, Temporelli PL, Lucci D, et al. Current management and treatment of patients with stable coronary artery diseases presenting to cardiologists in different clinical contexts: a prospective, observational, nationwide study. Eur J Prev Cardiol 2018;25:43-53.

62. Zito GB, Marzilli M, Orsini E, et al. Valutazione della qualità di vita e della prognosi dei pazienti con angina stabile trattati in accordo alle linee guida dai cardiologi ambulatoriali A.R.C.A. (Associazioni Regionali Cardiologi Ambulatoriali). Registro italiano multicentrico, prospettico, osservazionale. A.R.C.A. The ARCA Registry for Chronic Angina. Cardiologia AmbulatorialeOutpatient Cardiology: Fascicolo 2017;2:134-40. 